\title{
Use of emerging testing technologies and approaches for SARS-CoV-2: review of literature and global experience in an Australian context
}

\author{
Maryza Graham $^{1,2}$, Susan A. Ballard ${ }^{1}$, Shivani Pasricha ${ }^{3}$, Belinda Lin ${ }^{1}$, \\ Tuyet Hoang ${ }^{1,3}$, Timothy Stinear ${ }^{3}$, Julian Druce ${ }^{4}$, Mike Catton ${ }^{4}$, \\ Norelle Sherry ${ }^{1,5}$, Deborah Williamson ${ }^{1,3,5}$, Benjamin P. Howden ${ }^{1,3}$ \\ ${ }^{1}$ Microbiological Diagnostic Unit, Department of Microbiology and Immunology, The Uni- \\ versity of Melbourne at The Peter Doherty Institute for Infection and Immunity, Melbourne, \\ Vic, Australia; ${ }^{2}$ Department of Microbiology and Infectious Diseases, Monash Health, \\ Clayton, Vic, Australia; ${ }^{3}$ Department of Microbiology and Immunology, The University of \\ Melbourne at The Peter Doherty Institute for Infection and Immunity, Melbourne, Vic, \\ Australia; ${ }^{4}$ Victorian Infectious Diseases Reference Laboratory, Melbourne Health at The \\ Peter Doherty Institute for Infection and Immunity, Melbourne, Vic, Australia; ${ }^{5}$ Department \\ of Microbiology, Royal Melbourne Hospital, Parkville, Vic, Australia
}

\begin{abstract}
Summary
Emerging testing technologies for detection of SARS-CoV2 include those that are rapid and can be used at point-ofcare (POC), and those facilitating high throughput laboratory-based testing. Tests designed to be performed at POC (such as antigen tests and molecular assays) have the potential to expedite isolation of infectious patients and their contacts, but most are less sensitive than standardof-care reverse transcription polymerase chain reaction (RT-PCR). Data on clinical performance of the majority of emerging assays are limited with most evaluations performed on contrived or stored laboratory samples. Further evaluations of these assays are required, particularly when performed at POC on symptomatic and asymptomatic patients and at various time-points after symptom onset.

A few studies have so far shown several of these assays have high specificity. However, large prospective evaluations are needed to confirm specificity, particularly before the assays are implemented in low prevalence settings or asymptomatic populations. High throughput laboratorybased testing includes the use of new sample types (e.g., saliva to increase acceptability) or innovative uses of existing technology (e.g., sample pooling). Information detailing population-wide testing strategies for SARSCOV-2 is largely missing from peer-reviewed literature. Logistics and supply chains are key considerations in any plan to 'scale up' testing in the Australian context.

The strategic use of novel assays will help strike the balance between achieving adequate test numbers without overwhelming laboratory capacity. To protect testing of high-risk populations, the aims of testing with respect to the phase of the pandemic must be considered.
\end{abstract}

Key words: Testing; COVID-19; SARS-CoV-2.

Received 3 May, revised 12 July, accepted 3 August 2021

Available online 10 August 2021

\section{INTRODUCTION}

As the COVID-19 pandemic has progressed and diagnostic needs have evolved, a range of emerging testing technologies for detection of SARS-CoV-2 have become available. These include rapid diagnostic and point-of-care (POC) tests and those facilitating high-throughput laboratory-based testing. The quality of the evaluations for emerging tests are variable and often insufficient, especially for performance related to the intended use. ${ }^{1-3}$ The majority of available information regarding emerging tests and testing strategies is derived from high-prevalence scenarios and the relevance to the Australian context is unclear. We summarise the currently available information for emerging testing strategies and technologies to elucidate their potential use in the Australian context.

\section{IDENTIFYING TESTING APPROACHES USING MODELLING}

The characteristics of emerging assays relative to standard laboratory-based reverse transcription polymerase chain reaction (RT-PCR) vary considerably with respect to performance, turnaround time (TAT) and throughput (Table 1). Mathematical modelling studies examining the relative contributions of various testing strategies show that approaches with high test numbers and short TAT have the greatest impact. $^{4-6}$

Modelling has shown that minimising testing delay had the largest impact on reducing onward transmissions and optimising testing coverage further enhanced contact tracing effectiveness. ${ }^{4}$ Keeping the time between symptom onset and testing and isolation of an index case at 2 days or less is imperative for success in reducing the reproductive number, noting that rapid testing of symptomatic people is at least as important as the efficiency of contact tracing. ${ }^{4}$ Weekly PCR screening of healthcare workers (HCWs) and other high-risk groups, irrespective of symptoms was estimated to reduce their contribution to SARS-CoV-2 transmission (R) by $23 \%$. 
Table 1 Comparison of characteristics of emerging assays relative to standard laboratory-based RT-PCR

\begin{tabular}{|c|c|c|c|c|c|c|c|c|}
\hline Assay type & Available literature ${ }^{a}$ & TAT & Sensitivity & Specificity & Ease of use at POC & Scalability & Cost & Supply chain \\
\hline Standard laboratory-based RT-PCR & $+++/++++$ & Hours & ++++ & ++++ & $\mathrm{n} / \mathrm{a}$ & +++ & +++ & $++/+++$ \\
\hline \multicolumn{9}{|l|}{ 1. Innovations of molecular assays } \\
\hline Rapid or near POC RT-PCR & ++++ & Under 1 hour & ++++ & ++++ & ++ & + & ++++ & $+/++$ \\
\hline POC NAAT & $+/++$ & Minutes & $+++/++++$ & ++++ & ++++ & + & ++++ & $+/++$ \\
\hline Extraction-free LAMP & $+/++$ & Minutes-hours & $++/+++$ & $+++/+++$ & + & ++ & + & +++ \\
\hline Saliva RT-PCR & $+/++$ & Hours & $++/+++$ & ++++ & $\mathrm{n} / \mathrm{a}$ & +++ & +++ & +++ \\
\hline Pooling & ++ & Hours & +++ & ++++ & $\mathrm{n} / \mathrm{a}$ & ++++ & + & ++ \\
\hline Extraction-free RT-PCR & + & Hours & +++ & ++++ & $\mathrm{n} / \mathrm{a}$ & +++ & ++ & ++ \\
\hline RT-PCR with WGS & - & Hours-days & - & - & - & $-/++++$ & ++++ & - \\
\hline \multicolumn{9}{|l|}{ 2. Non-molecular assays } \\
\hline POC Antigen & $+/+++$ & Minutes & $++/+++$ & +++ & $+++/++++$ & ++ & + & $-/++$ \\
\hline \multicolumn{9}{|l|}{ 3. Emerging technology } \\
\hline CRISPR & + & Hours & +++ & +++ & - & - & + & - \\
\hline Microfluidics & - & Hours & $-1+$ & - & - & - & - & - \\
\hline Virolens & - & Seconds & - & - & - & - & - & - \\
\hline
\end{tabular}

-, unknown/insufficient data; +, minimal; ++, moderate; +++, high; ++++, very high; n/a not applicable; CRISPR, clustered regularly interspaced short palindromic repeats; LAMP, loop-mediated isothermal amplification; NAAT, nucleic acid amplification techniques; POC, point-of-care; RT-PCR, reverse-transcription polymerase chain reaction; TAT, turnaround time; WGS, whole genome sequencing.

${ }^{\text {a }}$ Peer-reviewed literature or independent evaluation of clinical performance.

This reduction is in addition to the reductions achieved when people self-isolate following symptoms, assuming test results are available at 24 hours. A $15 \%$ reduction in test sensitivity reduces the effectiveness of weekly screening from $23 \%$ to $19 \%$. If $80 \%$ of cases and contacts are identified and there is immediate testing following symptom onset and quarantine of contacts occurs within 24 hours, R can be reduced by $26 \%$. This result is on top of reductions achieved by self-isolating following symptoms. For 50\% coverage and a 48-hour delay in quarantining, the reduction in transmission is just $8 \%$.

One model found that effective screening depends largely on testing frequency and the speed of reporting and is only marginally improved by high test sensitivity. ${ }^{6}$ A notable assumption in this model is that during the exponential growth phase of the virus, the time between $10^{3}$ and $10^{5}$ copies $/ \mathrm{mL}$ is short, allowing a limited window in which only the more sensitive test could diagnose individuals. A limitation of this study is it did not consider test specificity: specificity considerations must be taken into account given that, for example, if a test is $80 \%$ sensitive and $98 \%$ specific, at $1 \%$ prevalence the positive predictive value would only be $28.8 \%$.

It has been suggested that people would possibly use negative low-sensitivity test results (conceivably falsely negative) to justify abandoning more proven interventions, such as wearing a mask and social distancing. It is probable that people who have high levels of SARS-CoV-2 in their respiratory secretions are more likely to be infectious than those with low levels, but whether lower-sensitivity tests can reliably detect persons who are likely to be infectious remains to be proven. It is clear that samples with lower PCR cycle threshold $(\mathrm{Ct})$ values are more likely to contain SARS-CoV-2 that can be detected in viral culture, but there is no clear separation among samples that are culture positive or not when using the $\mathrm{Ct}$ value. Furthermore, there remain no robust clinical data linking viral quantity to transmissibility.

Fundamentally, optimisation of SARS-CoV-2 testing is paramount to controlling the pandemic. Here we summarise the current literature for emerging testing technologies and approaches and discuss their potential in the Australian context. We have included peer-reviewed literature, independent evaluations and selected widely-cited pre-prints.

\section{INNOVATIONS IN MOLECULAR ASSAYS}

\section{Commercial point-of-care (POC) or near-POC molecular assays}

Commercial POC rapid nucleic acid amplification techniques (NAATs) fall into two categories: those with instrumentation widely available in Australia and those where instrumentation is less available (Table 2). Of the former group, the Xpert Xpress SARS-CoV-2 assay has shown reliable performance but has had severe limitations on test cartridge supply. Therefore, there is a need to investigate the performance of alternative assays for which there are less available data.

A March 2021 Cochrane review $^{8}$ found that sensitivities of rapid molecular assays varied according to test brand. Most of the data relate to the ID NOW COVID-19 (Abbott) and Xpert Xpress (Cepheid) assays. Using data from evaluations following the instructions for use (IFU), the average sensitivity of ID NOW was $73.0 \%$ [95\% confidence interval (CI) 66.8-78.4\%] and average specificity $99.7 \%$ (95\% CI 98.7-99.9\%). For Xpert Xpress, the average sensitivity was $100 \%(95 \%$ CI $88.1-100 \%)$ and average specificity $97.2 \%$ (95\% CI 89.4-99.3\%). A Foundation for Innovative New Diagnostics (FINDdx) independent evaluation determined the Xpert assay had $100 \%$ sensitivity and $99 \%$ specificity when compared to the Cobas assay (Roche) which is widely used in Australia.

The variable performance in sensitivity of the ID NOW SARS-CoV-2 assay may be attributed to the type of swab used, the time of testing following onset of symptoms and the reference assay used in the different studies. ${ }^{10-14}$ Several of the early evaluations of ID NOW used viral transport media/universal transport media rather than dry swabs, so did not follow the manufacturer's IFU, thus requiring further evaluation. 
Table 2 Rapid or near POC nucleic acid amplification tests (as of 12 April 2021)

\begin{tabular}{|c|c|c|}
\hline Assay & FDA Reference panel LOD (NDU/mL) & Approximate turnaround time \\
\hline \multicolumn{3}{|l|}{ TGA listed } \\
\hline Cepheid Xpert Xpress SARS-CoV-2/Flu/RSV & $\mathrm{n} / \mathrm{a}$ & \\
\hline BioFire FilmArray Respiratory Panel 2.1plus SARS-CoV-2 assay & 6000 & $45 \min ^{111}$ \\
\hline Abbott ID NOW COVID-19 & 300,000 & $15-20 \mathrm{~min}$ \\
\hline Roche Cobas SARS-CoV-2 and Influenza A/B Liat & 5400 & $20 \mathrm{~min}$ \\
\hline Sansure 2019-nCoV Nucleic Acid Diagnostic Kit & Did not provide shipping information & $30 \mathrm{~min}$ \\
\hline USTAR EasyNat Diagnostic Kit & $\mathrm{n} / \mathrm{a}$ & $79 \min$ \\
\hline \multicolumn{3}{|l|}{ Not TGA listed (FDA approved/CE marked) } \\
\hline Credo SARS-CoV-2 VitaPCR & $\mathrm{n} / \mathrm{a}$ & $20 \mathrm{~min}$ \\
\hline Aries SARS-CoV-2 Assay & 180,000 & \\
\hline Accula SARS-CoV-2 & Under interactive review & $30 \min$ \\
\hline Talis One COVID-19 Assay & $\mathrm{n} / \mathrm{a}$ & $30 \mathrm{~min}$ \\
\hline Visby Medical Instrument-free PCR & 54,000 & $30 \mathrm{~min}$ \\
\hline Atila BioSystems iAMP & 180,000 & $\sim 1$ hour from dry swab to final result \\
\hline Cue COVID-19 Test & 60,000 & $20 \mathrm{~min}$ \\
\hline T2 Biosystems T2SARS-CoV-2 Panel & 18,000 & $\begin{array}{l}\text { Results in }<2 \mathrm{~h} \text {; throughput up to } 60 \\
\text { samples/day }\end{array}$ \\
\hline Quidel Solana SARS-CoV-2 assay & $\mathrm{n} / \mathrm{a}$ & \\
\hline Pro-AmpRT SARS-CoV-2 test & Did not provide shipping information & \\
\hline Color Genomics SARS-CoV-2 RT-LAMP Diagnostic Assay & 18,000 & \\
\hline Seasun AQ-TOP COVID-19 Rapid Detection Kit & 6000 & $\begin{array}{l}\sim 1 \mathrm{~h} \text { ('fast extraction' of } \sim 5 \text { min plus } \\
30 \text { min for detection) }\end{array}$ \\
\hline Lucira & $\mathrm{n} / \mathrm{a}$ & \\
\hline COVIDNudge & $\begin{array}{l}\text { Sensitivity } 94 \%(95 \% \text { CI } 86-98) \\
\text { Specificity } 100 \%(99-100)^{112}\end{array}$ & $90 \mathrm{~min}$ \\
\hline SAMBA II & $\mathrm{n} / \mathrm{a}$ & $<90 \mathrm{~min}$ \\
\hline OptiGene & $\mathrm{n} / \mathrm{a}$ & $<20$ min \\
\hline Novodiag & $\mathrm{n} / \mathrm{a}$ & $1 \mathrm{~h} 20 \mathrm{~min}$ \\
\hline POCKIT Central & $\mathrm{n} / \mathrm{a}$ & $85 \mathrm{~min}$ \\
\hline
\end{tabular}

n/a, not applicable; POC, point-of-care; RT-PCR, reverse-transcription polymerase chain reaction.

To allow comparison of analytical performance of different molecular assays, the US Food and Drug Administration (FDA) developed a SARS-CoV-2 Reference Panel. ${ }^{15}$ Results of various concentrations of inactivated SARS-CoV-2 as blinded samples for a number of different POC molecular assays are shown in Supplementary Table 1 (Appendix A). Other TGA listed POC or near-POC assays with significant performance data available in peer-reviewed literature and showing similar performance to Xpert assay include the Liat (Roche) and the FilmArray (BioFire). ${ }^{16,17}$

\section{Loop-mediated isothermal amplification (LAMP)}

LAMP was developed as a rapid and cheap method to amplify DNA/RNA target at a single reaction temperature, bypassing the need for sophisticated thermal cycling equipment. Several studies have evaluated various novel LAMP methods for SARS-CoV-2 using extraction-free methods, with results available within minutes. ${ }^{18-26}$ Extraction of RNA improves sensitivity approaching that of laboratory-based RT-PCR but increases testing complexity, TAT and the need for extraction reagents, which at times during the pandemic have been in short supply. This rapid assay can potentially be used at POC with medium throughput capability. ${ }^{22}$ Currently, there are limited studies on LAMP at POC; therefore, its feasibility, particularly with and without an extraction step, requires further study.

\section{Saliva testing}

Collecting saliva specimens for SARS-CoV-2 testing has several advantages over nasopharyngeal swab (NPS): less patient discomfort, is amenable to self-collection and poses less risk to HCWs. ${ }^{27}$ There are 17 US FDA Emergency Use Authorisation assays for SARS-CoV-2 RNA detection in saliva: four are authorised for use in asymptomatic populations. The limit of detection (LOD) for evaluated assays ranges from $600 \mathrm{NDU} / \mathrm{mL}$ to $180,000 \mathrm{NDU} / \mathrm{mL}$ in FDA Reference Panel data, ${ }^{15}$ but limited clinical performance evaluations are available. There are currently three TGA approved COVID-19 tests (NeumoDx, PerkinElmer and Seegene) for use with saliva, meaning that most Australian laboratories are required to perform their own validation of assays for saliva testing.

Six published meta-analyses using various RT-PCR assays have generally found saliva testing to be less sensitive than testing of nasopharyngeal swabs. ${ }^{27-32}$ Studies using saliva testing for SARS-CoV-2 detection have employed various collection methods, processing methods, assays and patient populations and therefore have yielded variable results. ${ }^{33-39}$

Two studies testing saliva samples used the Xpert assay. ${ }^{40,41}$ In one, there was good correlation with validated assays in a mix of emergency department (ED), inpatients and outpatients with positive agreement (PPA) of 98.9\% (87/ 88 specimens) and negative agreement (NPA) of $100 \% .^{40}$ The other found good correlation with paired NPS in ED 
and suspected COVID-19 patients, with a PPA of 96\% (47/49 patients) and NPA of $99 \%$. $^{41}$

Given the advantages of saliva testing, and as several reasonably sized studies show moderately high sensitivity, further studies are warranted to evaluate the optimum specimen collection and processing methods for saliva testing. In the interim, the current Australian Testing Framework (updated 25 June 2021) states that Australian laboratories are using saliva samples for RT-PCR to facilitate expanded surveillance, but that saliva sample testing is not intended to replace well validated, gold-standard swab-based RT-PCR for diagnosis of disease in symptomatic people. ${ }^{42}$

Currently there are very few clinical validation studies on the use of saliva, as sample material for rapid antigen tests and data on the sensitivity of the tests are lacking. ${ }^{43}$ Limited available information suggests poor sensitivity compared to nasal swabs. ${ }^{44}$

\section{Sample pooling}

Testing pooled specimens rather than individual testing saves on resources and time. ${ }^{45,46}$ The European Centre for Disease Prevention and Control (ECDC) guidelines suggest largescale population-wide testing by means of pool-based strategies. ${ }^{45,47}$ The disease prevalence in a population affects the efficiency of pooled testing strategies, with lower prevalence potentially enabling a larger pool size. The US Centers for Disease Control and Prevention (CDC) found that NAAT for SARS-CoV-2 reliably returned a positive result when one positive sample was mixed with four negatives and could reduce the number of tests needed by $>50 \%$ in scenarios such as COVID-19 prevalence of $<5 \%$. ${ }^{48}$ As pooled specimens are diluted when the pool is larger, there is a higher likelihood of generating false-negative results so monitoring prevalence is also important to limit this. ${ }^{49}$

\section{Extraction-free RT-PCR}

The extraction step of RT-PCR improves sensitivity by purifying DNA/RNA. The advantages of extraction-free PCR are that it is rapid, cost-effective and not reliant on extraction reagents that have been in short supply. The main disadvantage of extraction-free RT-PCR is reduction in sensitivity, which ranges between 81.3 and $94.6 \% .^{50-52}$

\section{RT-PCR with whole-genome sequencing (WGS)}

WGS of SARS-CoV-2 can improve the resolution of outbreak clusters. It can better define possible transmission networks and therefore inform public health responses. Combining diagnostic testing with WGS has the potential to improve TAT for the genomics results since high throughput detection of SARS-CoV-2 can be combined with WGS of positive samples such as with COVIDSeq assay (Supplementary Table 2, Appendix A). ${ }^{53-56}$

\section{ANTIGEN TESTS}

There is a paucity of peer-reviewed literature evaluating the clinical performance of the various antigen assays, particularly in low-prevalence settings (Table 3). A 2020 Cochrane review found the sensitivity of included antigen tests (Beijing Savant, Coris Bioconcept, Liming Bio, RapiGEN, Shenzhen Bioeasy and one in-house assay) varied considerably across studies. Based on five studies ( $n=943)$, the average sensitivity was $56.2 \%$ (95\% CI $29.5-79.8 \%)$ and average specificity was $99.5 \%$ (95\% CI 98.1-99.9\%). ${ }^{8}$ Data for individual antigen tests were limited, with no more than two studies for any test. This review identified that early evaluations of POC tests are largely based on remnant laboratory samples. It is uncertain whether tests will perform similarly in clinical practice, and with regards to symptom type, duration of symptoms, or in asymptomatic people. ${ }^{8}$

A March 2021 update to the Cochrane review found that estimates of antigen sensitivity varied considerably between studies and between brands. There were differences between symptomatic $(72.0 \%, 95 \%$ CI 63.7-79.0\%) and asymptomatic participants $(58.1 \%, 95 \%$ CI $40.2-74.1 \%)$. Average sensitivity was higher in the first week after symptom onset $(78.3 \%, 95 \%$ CI $71.1-84.1 \%)$ than in the second week of symptoms $(51.0 \%, 95 \%$ CI $40.8-61.0 \%)$. Using data from manufacturer's instructions for use (IFU) compliant evaluations in symptomatic participants, summary sensitivities ranged from 34.1\% (95\% CI 29.7-38.8\%; Coris Bioconcept) to $88.1 \%$ (95\% CI 84.2-91.1\%; SD Biosensor STANDARD Q). Average specificities were high in symptomatic and asymptomatic participants, and for most brands (overall summary specificity $99.6 \%, 95 \%$ CI $99.0-99.8 \%) .{ }^{8}$

Of the 13 TGA approved antigen tests, the Panbio COVID$19 \mathrm{Ag}$ rapid test (Abbott) currently has the most peerreviewed literature, including for children. ${ }^{57-68}$ Two early studies of the Panbio COVID-19 Ag rapid test found sensitivity between $73-81 \%$ and $100 \%$ specificity. ${ }^{61,62}$ In addition, FINDdx independent evaluations found Panbio sensitivity for NPS in symptomatic persons to be $85.5 \%(95 \%$ CI $78.2-90.6 \%$ ) with clinical specificity of $100 \%$ (95\% CI 99.1-100\%). ${ }^{9}$ An Australian multi-site assessment of the Panbio antigen test in a low-prevalence setting found a specificity of $99.96 \%$ in 2413 individuals $(95 \%$ CI $99.73-100 \%){ }^{69}$

Of the other TGA approved antigen tests, the SD Biosensor Standard Q (supplied in Australia by Roche Diagnostics) also has a significant amount of peer-reviewed literature. ${ }^{66,68,70-}$ ${ }^{76}$ FINDdx evaluation found sensitivities for NPS in symptomatic persons between $88.7-89 \%$ with specificity $97.6-99.7 \%$. $^{9}$

Continuously emerging results from FINDdx independent evaluations are likely to substantially inform performance in real-life scenarios (Table 4). ${ }^{9}$ For example, recent FINDdx results comparing performance of nasal swabs (NS) to NPS found for the the PanBio antigen test a sensitivity of $86.4 \%$ (95\% CI $73.3-93.9 \%)$ for NS and $90.9 \%$ (95\% CI $78.8-96.4 \%$ ) for NPS in a small study with 44 positive cases; for SD Biosensor a sensitivity of $80.5 \% \quad(95 \%$ CI 66.0-89.8\%) for NS and 73.2\% (95\%CI 58.1-84.3\%) for NPS in 41 positive patients; and for NowCheck a sensitivity of $89.9 \%$ (95\% CI $81.3-94.8 \%$ ) for both NS and NPS in 79 positive patients.

\section{OTHER NOVEL EMERGING TECHNOLOGIES CRISPR}

Two assays for detection of SARS-CoV-2 RNA combining isothermal amplification and CRISPR technology have been approved by the US FDA: SHERLOCK and DETECTR (Mammoth Biosciences). The SHERLOCK assay is portable, and testing can be performed on a heat block or water bath. CRISPR assay reagents can cost $<\$ 1$ per test. $^{77}$ 
Detection can be performed by lateral flow assay (portable, read by eye) or fluorescence. The reaction TAT for the SHERLOCK assay is 1 hour, whereas it is 45 minutes for the DETECTR assay. According to the IFU, the DETECTR assay LOD is 12,000 copies/mL UTM. The SHERLOCK assay was included in the recent FDA Reference Panel testing and LOD was found to be $6000 \mathrm{NDU} / \mathrm{mL}$, which was more sensitive than the CDC assay (Supplementary Table 1, Appendix A).

Overall, available information suggests CRISPR may be less sensitive when compared to most commercial RT-PCR.
However, studies have shown specificity between $71.4 \%$ and $98.5 \%$ for CRISPR, ${ }^{78,79}$ so further evaluations are required since a recent review has concluded that although CRISPR technology is still in its infancy it is potentially revolutionary as tests are rapid and portable. ${ }^{80}$

\section{Microfluidics}

Currently there is one FDA approved assay for SARS-CoV2 detection in saliva using microfluidics: Advanta Dx (Fluidigm Corporation). Saliva is a validated sample type

Table 3 Peer-reviewed literature for TGA listed SARS-CoV-2 antigen tests (as of 12 April 2021)

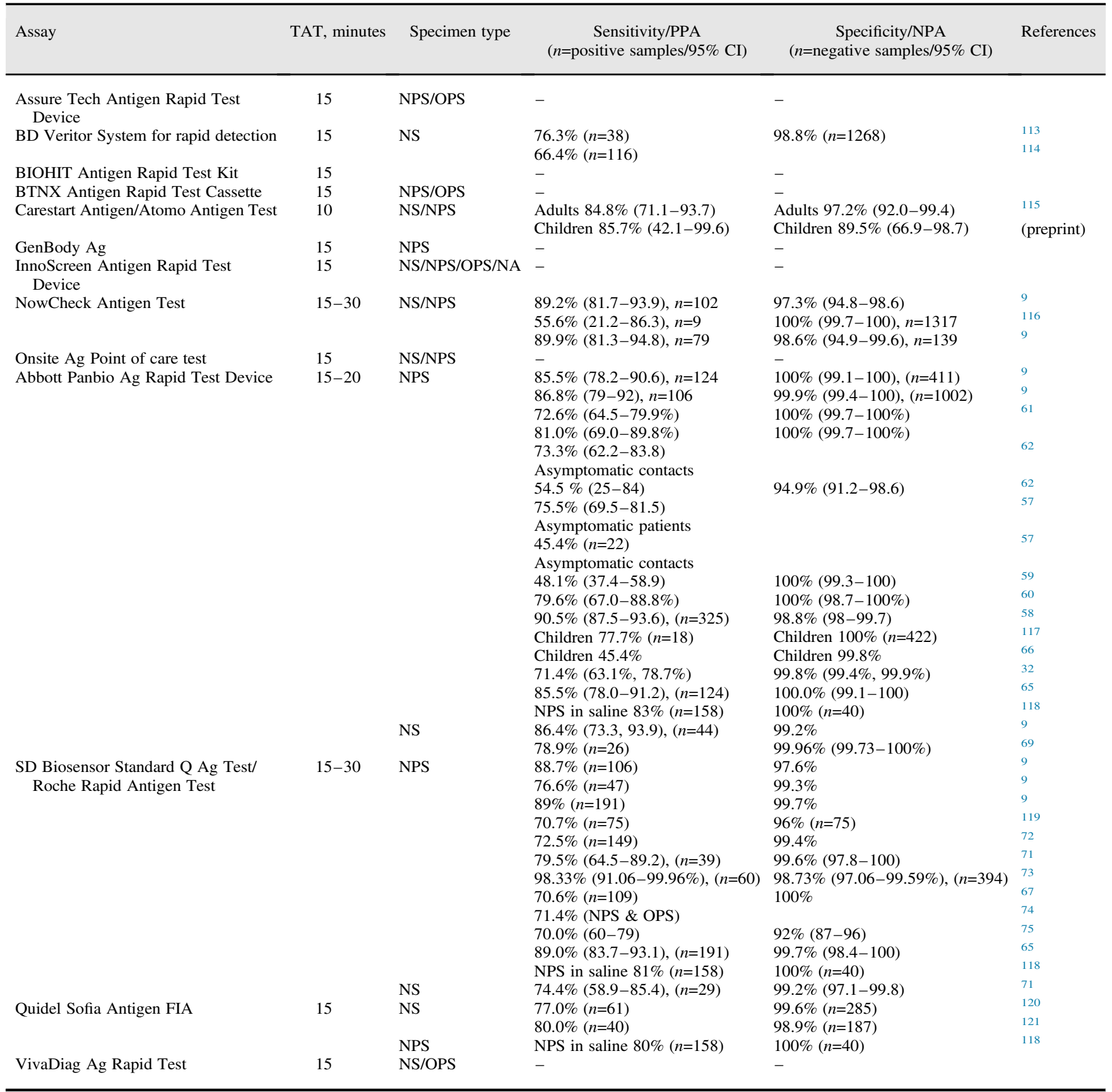

NA, nasal aspirate; NPA, negative agreement; NPS, nasopharyngeal swab; NS, nasal swab; OPS, oropharyngeal swab; PPA, positive agreement; TAT, turnaround time; $95 \%$ CI, $95 \%$ confidence interval.

Nil peer-reviewed literature in PubMed or independent evaluation in FINDdx or FDA reference panel. 
Table 4 Foundation for Innovative New Diagnostics (FIND) independent evaluations for TGA listed antigen assays (as of 14 April 2021)

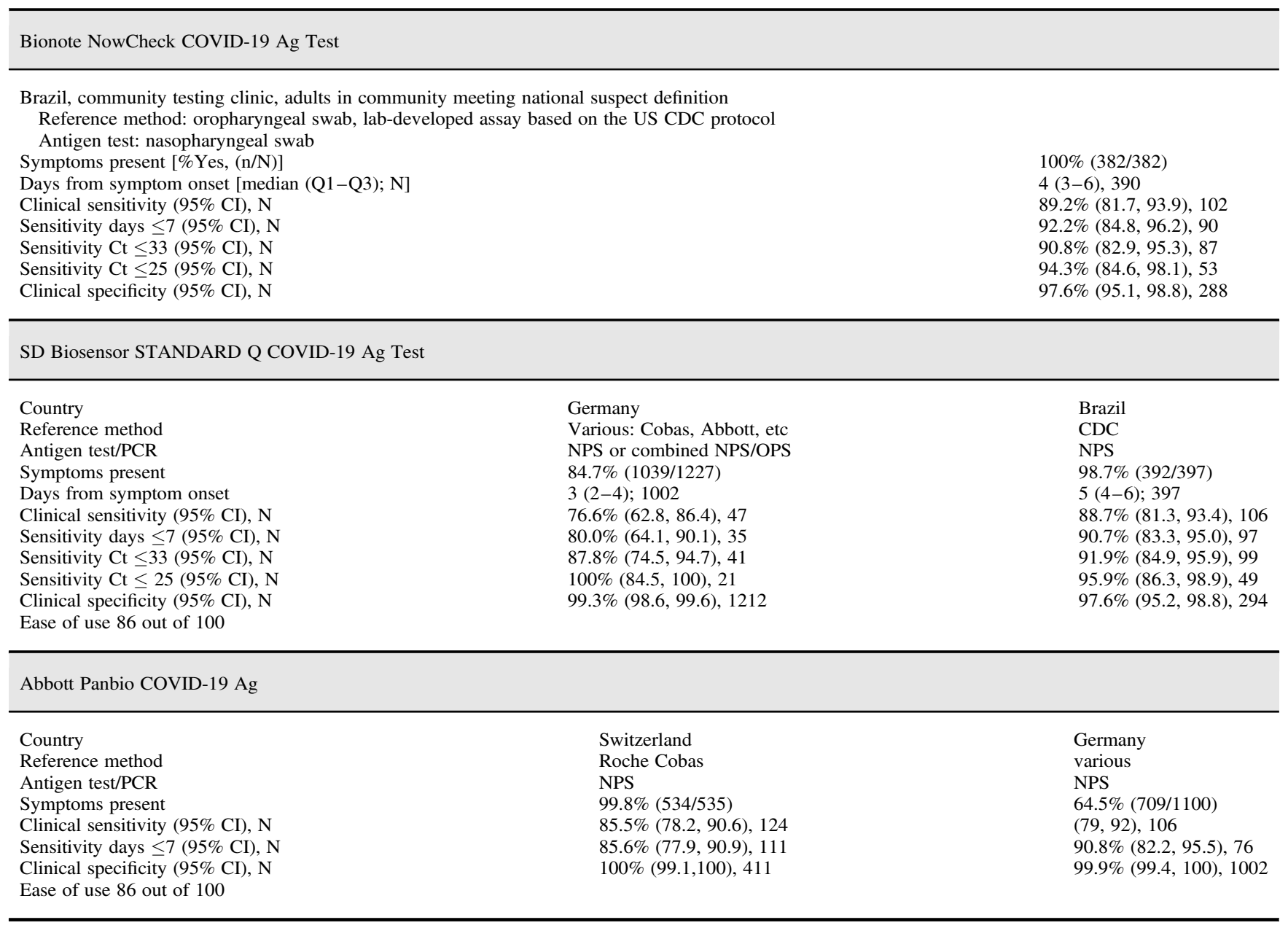

CDC, Centers for Disease Control; NPS, nasopharyngeal swab; OPS, oropharyngeal swab.

and the test duration is 2 hours 32 minutes. No published evaluations are currently available. The LOD was 54,000 NAAT detectable units (NDU)/mL in the FDA Comparative Data (Supplementary Table 1, Appendix A).

\section{Virolens}

The Virolens system uses a self-administered mouth swab, which is placed inside a cartridge and inserted into a portable tabletop device. Inside the system is a holographic microscope designed to look at nano-scale structures and the light diffracted off the surface of each cell in the sample. This data is run through a computer trained by artificial intelligence to identify the unique pattern of the virus from other cells. The Virolens system is self-contained and can give a result in 20 seconds. Following the first round of field testing carried out in partnership with London Heathrow Airport, Virolens is about to embark on clinical trials (including Heathrow Airport and Leidos in the USA). ${ }^{81}$ The UK Medicines and Healthcare products Regulatory Agency have approved the device and submission has been made to the TGA, however no peer-reviewed performance data are publicly available. Ultra-rapid tests with novel mechanisms of action such as this test may have a unique role in the pandemic, subject to performance evaluations.

\section{Breathalysers}

Several countries are at various stages of large clinical evaluations of instruments for breath detection of SARSCoV-2 infection: examples include GeNose, BreathPass, SpiroNose, Brethonix. ${ }^{82-90}$ Detection technologies employed include gas chromatography, different forms of mass spectrometry, and nanosensors to either detect volatile organic compounds or directly detect the virus. ${ }^{91}$ There is a paucity of peer-reviewed literature evaluating large-scale clinical performance of the various assays. In May 2021 Singapore's Health Sciences Authority (HSA) granted provisional authorisation for two COVID-19 breathalysers. One of these, the TracieX Breathalyser is reported to have a sensitivity of $95 \%$ and specificity of $97.8 \%$ and the HSA will continue to closely monitor the clinical performance of these tests as they are deployed locally. ${ }^{92}$ However, another Dutch assay was found to have lower specificity of $82 \%$ when tested on asymptomatic persons and not long after it was launched its use was halted by Amsterdam's Public Health Service after a concern about sensitivity. ${ }^{93,94}$ Assays with good performance would have the potential to rapidly screen large populations as these assays screen individuals within minutes, but assays with high specificity would be more useful in low-prevalence settings such as Australia. 


\section{Trained sniffer dogs}

A March 2021 World Health Organization (WHO) blueprint has reviewed the global use of sniffer dogs for SARS-CoV-2 detection and found variable sensitivity between $65.4-100 \%$ and specificity between $85.2-98 \%$. The blueprint describes that one dog is able to screen $250-300$ persons a day. Challenges include the need for standardisation and validation of approaches and difficulties in training dogs in lowprevalence settings. $^{95}$

\section{Environmental testing}

Researchers in the Netherlands, France and the USA have demonstrated a correlation between wastewater SARS-CoV-2 concentrations and clinical cases. ${ }^{96}$ Some of the results suggested that the RNA concentrations could provide advanced notice of infections (4-7 days) before confirmed cases ${ }^{96}$ and retrospective testing has shown that SARS-CoV-2 is present several months before large outbreaks. ${ }^{97}$ There is potential to use environmental surveillance for early warning of outbreaks in countries that have already contained transmission but this may be challenged by detection of viral RNA shedding from past infection. In the first confirmed detection of SARS-CoV2 in untreated wastewater in the Australian community, two positive detections of SARS-CoV-2 RNA were found within a 6-day period from the same wastewater treatment plant. ${ }^{98}$ Estimated RNA copy numbers in the wastewater were then used to estimate the number of infected individuals in the catchment via Monte Carlo simulation. As the proportion of infected patients shedding viral RNA in stool is subject to substantial variation $(27 \%$ in a Chinese cohort, $88 \%$ in a German cohort) and given the uncertainty and variation in other input parameters, the model estimated a median range of $171-1090$ infected people in the catchment. ${ }^{98}$ In view of these limitations, the authors concluded that one of the biggest challenges is to establish predictions from the sewage RNA concentrations to the actual case numbers in the community.

There is a paucity of clinical studies of air sampling for SARS-CoV-2 detection: for example, one recent preprint comparing a commercial air sampler (not TGA listed) to other environmental testing in 32 hospital rooms of COVID-19 positive patients found that among positive rooms, 32\% had only active air samples that returned positive results, while $\sim 27 \%$ and $\sim 9 \%$ had only one or more surface swabs or passive settling plates that returned a positive result respectively; $32 \%$ of rooms had more than one sample type that returned a positive result. ${ }^{99}$ Therefore, the utility of air sampling in various prevalence contexts relevant to Australia requires further research. ${ }^{99}$

\section{POC ANTIBODY TESTS}

The Peter Doherty Institute for Infection and Immunity has so far evaluated 23 different serology-based point of care tests with results on the TGA website. This validation suggests that manufacturers have claimed a better sensitivity compared to that observed in the Doherty Institute studies (when compared to a molecular-based method). The Doherty Institute studies did demonstrate that the sensitivity of most tests improved with increasing duration (i.e., longer time) between sample collection and symptom onset, up to approximately 20-30 days post-symptom onset. ${ }^{100}$ The Australian Testing Framework indicates that POC serology tests are not recommended as first line tests for the diagnosis of acute infection and that the role of these tests is uncertain in the context of Australia's broadly low prevalence setting. These recommendations may change with changing prevalence, vaccination rates and border opening.

\section{CONSIDERATIONS FOR 'OUT OF LAB' OR POC TESTING}

POC testing may be located at the bedside or may be 'near' POC and performed in a mobile laboratory (e.g., laboratory in a van) or in a 'pop-up' laboratory that can be in a marquee or a local room. For mobile laboratories, the effects of transportation and weather extremes on equipment performance need to be considered. Biosafety requirements will depend on the assay used: biosafety cabinets may be required (in addition to personal protective equipment) particularly if virus in samples is not inactivated or if testing is performed in 'open' instrumentation or may generate aerosols.

Some assay manufacturer's instructions require a short time (e.g., 15 minutes) between sample collection and processing and this will influence the location of POC testing. Some assays are easier to use than others and the number of sample manipulation steps required will also impact testing location, the required training and qualifications of staff.

In-field recording and reporting of results may require dedicated equipment for sample accessioning and result management with capacity to integrate remote devices with laboratory information systems that can facilitate reporting of results to patients, requesting practitioners and public health units.

\section{OVERSEAS EXPERIENCE AND TESTING STRATEGIES}

Information detailing population-wide testing strategies for SARS-COV-2 is largely missing from the literature. Overall, a variety of strategies are being considered overseas and the relevance of these to the Australian context is unclear.

Strategies used in high prevalence settings include a Liverpool testing pilot of over 3000 people using the Innova lateral flow antigen test which missed $60 \%$ of infected asymptomatic people, including $33 \%$ of those with high viral loads. Of note, Public Health England's evaluation of the Innova test showed that its sensitivity was $79.2 \%$ when used by trained laboratory scientists, $73 \%$ when used by trained healthcare staff, but only $57.5 \%$ when used by track and trace centre staff employed by the pharmacy chain Boots. ${ }^{101,102}$ Mass antigen testing (of the entire adult population) in Slovakia has generated controversy and a recent modelling study has concluded that while it was impossible to disentangle the precise contribution of infection control measures and mass testing, mass testing is likely to have had a substantial effect in curbing the pandemic. ${ }^{103,104} \mathrm{~A}$ hospital preprocedural surveillance PCR testing study in Seattle found low rates of positivity despite a community-wide outbreak. ${ }^{105}$

The European Centre for Disease Prevention and Control (ECDC) suggests that rapid antigen tests can contribute to overall COVID-19 testing capacity, especially in situations in which RT-PCR testing capacity is limited and the ECDC agrees with the minimum performance requirements set by the WHO at $\geq 80 \%$ sensitivity and $\geq 97 \%$ specificity. ${ }^{47,106}$ 
Strategies used in low-prevalence countries include rapid antigen test pre-event testing, recently introduced in Singapore for large gatherings such as tradeshows, live performances, spectator sports and weddings. ${ }^{107}$ The effect of this testing strategy will be of interest to other low-prevalence countries such as Australia. 108

The WHO implementation guide for SARS-CoV-2 rapid antigen tests suggests that appropriate scenarios for their use include outbreak or high-risk scenarios, particularly during widespread community transmission, but only limited use in low-prevalence populations or in asymptomatic individuals. ${ }^{109}$

Australia's testing strategy is guided by the Communicable Diseases Network Australia (CDNA)/Public Health Laboratory Network (PHLN) Testing Framework. The role of each test is dependent on the 'Epidemiological Zone' or phase of the pandemic which takes into account local prevalence and public health objectives of testing in that context. ${ }^{42}$ The role of diagnostic tests for symptomatic people (POC molecular, antigen and antibody tests) and screening tests for asymptomatic people (such as breathalysers, wastewater/sewerage surveillance or air sampling) will change depending on the presence and degree of local community transmission as this will affect the predictive value of these tests. For example, The Australian Testing Framework indicates that for Epidemiological Zone 1 (no community transmission) POC RTPCR tests may be useful in rural and remote communities when rapid TAT is required or that for Epidemiological Zone 3 (community transmission placing burden on response capacity) rapid antigen tests may prove useful as a screening test for individuals in high risk settings where the pre-test probability is high. The Framework recommends that positive antigen tests would require RT-PCR for confirmation. PHLN and CDNA also recommend reflex RT-PCR of suspected COVID-19 cases that return a negative rapid antigen test result. Although for some tests this may require the collection of two swabs, there are emerging data evaluating the performance of using the universal transport media from standard-of-care swabs in the antigen test buffer instead of collecting a second swab. ${ }^{110}$

As the pandemic evolves, with increasing vaccination rates and plans to open Australian borders, the roles of each test will evolve in the context of emerging performance data of the test in each prevalence context.

\section{THE FUTURE OF DIAGNOSTIC TEST DEVELOPMENT}

In Australia, the classification of assays as TGA listed or not (Table 2; Supplementary Table 3, Appendix A) will determine their availability for use in Australia. Assays that are not TGA listed but are available overseas, or that have received funding for development, are required to go through an approval process to ensure that minimum quality and performance requirements are met. Various similar processes are in place overseas (e.g., FDA approval for US or CE-marking for Europe). Due to differences in submissions from manufacturers and approval requirements this leads to varying availability of tests between countries.

For example, the US National Institutes of Health (NIH) awarded \$248.7 million in contracts to seven diagnostic companies under its first round of the Rapid Acceleration of COVID-19 Diagnostics Initiative. ${ }^{90}$ More than 650 applications were submitted and hundreds of experts from government, academia, and industry helped evaluate applications. NIH selected approximately 100 of the best concepts to enter a 1-week evaluation process. Thirty-one of these moved to a 4-6-week period of initial validation. The seven tests to receive this funding were the first to be chosen for scale-up and delivery to the marketplace (Supplementary Table 3, Appendix A). It is hoped that such initiatives may facilitate rapid development and approval of novel diagnostics to enable timely response during emergency scenarios such as the current COVID-19 pandemic.

\section{CONCLUSIONS}

New technologies for detection of SARS-CoV-2 are emerging at an unprecedented pace but reliable information on individual assay performance, availability and supply chains is highly variable. Large-scale evaluations of the performance of available assays in the context of intended use are required to inform optimal deployment for control of the pandemic in Australia.

Conflicts of interest and sources of funding: This work was supported by the Australian Government Department of Health Medical Research Future Fund (MRFF) COVID-19 diagnostics grant provided to the Peter Doherty Institute for Infection and Immunity. The authors state that there are no conflicts of interest to disclose.

\section{APPENDIX A. SUPPLEMENTARY DATA}

Supplementary data to this article can be found online at https://doi.org/10.1016/j.pathol.2021.08.001.

Address for correspondence: Dr Maryza Graham, The Peter Doherty Institute for Infection and Immunity, 792 Elizabeth St, Melbourne, Vic, 3000, Australia. E-mail: maryza.graham@unimelb.edu.au

\section{References}

1. Freire-Paspuel B, Vega-Mariño P, Velez A, et al. Evaluation of nCoV QS (MiCo BioMed) for RT-qPCR detection of SARS-CoV-2 from nasopharyngeal samples using CDC FDA EUA qPCR kit as a gold standard: an example of the need of validation studies. J Clin Virol 2020; 128: 104454 .

2. Wang X, Yao H, Xu X, et al. Limits of detection of 6 approved RT-PCR kits for the novel SARS-Coronavirus-2 (SARS-CoV-2). Clin Chem 2020; 66: 977-9.

3. Edson DC, Casey DL, Harmer SE, et al. Identification of SARS-CoV-2 in a proficiency testing program. Am J Clin Pathol 2020; 154: 475-8.

4. Kretzschmar ME, Rozhnova G, Bootsma MCJ, et al. Impact of delays on effectiveness of contact tracing strategies for COVID-19: a modelling study. Lancet Publ Health 2020; 5: e452-9.

5. Grassly NC, Pons-Salort M, Parker EPK, et al. Comparison of molecular testing strategies for COVID-19 control: a mathematical modelling study. Lancet Infect Dis 2020; 20: 1381-9.

6. Larremore DB, Wilder B, Lester E, et al. Test sensitivity is secondary to frequency and turnaround time for COVID-19 screening. medRxiv 2020; Sep 8: https://doi.org/10.1101/2020.06.22.20136309.

7. Pettengill MA, McAdam AJ. Can we test our way out of the COVID-19 pandemic? J Clin Microbiol 2020; 58: e02225-20.

8. Dinnes J, Deeks JJ, Adriano A, et al. Rapid, point-of-care antigen and molecular-based tests for diagnosis of SARS-CoV-2 infection. Cochrane Database Syst Rev 2021; 3: CD013705.

9. FIND. FIND evaluations of SARS-COV-2 assays. Cited 22 Apr 2021. https://www.finddx.org/covid-19/sarscov2-eval/

10. Rhoads DD, Cherian SS, Roman K, et al. Comparison of Abbott ID Now, DiaSorin Simplexa, and CDC FDA emergency use authorization methods for the detection of sars-cov-2 from nasopharyngeal and nasal swabs from individuals diagnosed with COVID-19. J Clin Microbiol 2020; 58: e00760-20. 
11. Basu A, Zinger T, Inglima K, Woo KM, et al. Performance of Abbott ID Now COVID-19 Rapid nucleic acid amplification test using nasopharyngeal swabs transported in viral transport media and dry nasal swabs in a New York City academic institution. J Clin Microbiol 2020 58. e01136-20.

12. Smithgall MC, Scherberkova I, Whittier S, Green DA. Comparison of Cepheid Xpert Xpress and Abbott ID now to Roche cobas for the rapid detection of SARS-CoV-2. J Clin Virol 2020; 128: 104428.

13. Mitchell SL, George KS. Evaluation of the COVID19 ID NOW EUA assay. J Clin Virol 2020; 128: 104429.

14. Harrington A, Cox B, Snowdon J, et al. Comparison of Abbott ID Now and Abbott m2000 methods for the detection of SARS-CoV-2 from nasopharyngeal and nasal swabs from symptomatic patients. J Clin Microbiol 2020; 58: e00798-20.

15. U.S. Food and Drug Administration. SARS-CoV-2 Reference Panel Comparative Data. 2020; cited 28 Sep 2020. https://www.fda.goy/ medical-devices/coronavirus-covid-19-and-medical-devices/sars-coy2-reference-panel-comparative-data

16. Hansen G, Marino J, Wang ZX, et al. Clinical performance of the point-of-care Cobas Liat for detection of SARS-CoV-2 in 20 minutes: multicenter study. J Clin Microbiol 2021; 59: e02811-20.

17. Creager HM, Cabrera B, Schnaubelt A, et al. Clinical evaluation of the BioFire ${ }^{\circ}$ respiratory panel 2.1 and detection of SARS-CoV-2. J Clin Virol 2020; 129: 104538.

18. Zhang Y, Odiwuor N, Xiong J, et al. Rapid molecular detection of SARS-CoV-2 (COVID-19) virus RNA using colorimetric LAMP. medRxiv 2020; Feb 29: https://doi.org/10.1101/2020.02.26.20028373.

19. Yu L, Wu S, Hao X, et al. Rapid colorimetric detection of COVID-19 coronavirus using a reverse tran-scriptional loop-mediated isothermal amplification (RT-LAMP) diagnostic platform. Clin Chem 2020; 66: $975-7$

20. Yang W, Dang X, Wang Q, et al. Rapid detection of SARS-CoV-2 using reverse transcription RT-LAMP method. medRxiv 2020; Mar 3 : https://doi.org/10.1101/2020.03.02.20030130.

21. Lu R, Wu X, Wan Z, et al. A novel reverse transcription loop-mediated isothermal amplification method for rapid detection of SARS-CoV-2 Int J Mol Sci 2020; 21: 2826.

22. Osterdahl MF, Lee KA, Lochlainn MN, et al. Detecting SARS-CoV-2 at point of care: preliminary data comparing loop-mediated isothermal amplification (LAMP) to polymerase chain reaction (PCR). BMC Infect Dis 2020; 20: 783.

23. Park GS, Ku K, Baek SH, et al. Development of reverse transcription loop-mediated isothermal amplification assays targeting Severe Acute Respiratory Syndrome Coronavirus 2 (SARS-CoV-2). J Mol Diagn 2020; 22: 729-35.

24. Yan C, Cui J, Huang L, et al. Rapid and visual detection of 2019 novel coronavirus (SARS-CoV-2) by a reverse transcription loopmediated isothermal amplification assay. Clin Microbiol Infect 2020; 26: 773-9.

25. Lee JYH, Best N, McAuley J, et al. Validation of a single-step, singletube reverse transcription loop-mediated isothermal amplification assay for rapid detection of SARS-CoV-2 RNA. J Med Microbiol 2020; 69: $1169-78$.

26. Lamb LE, Bartolone SN, Ward E, et al. Rapid detection of novel coronavirus/Severe Acute Respiratory Syndrome Coronavirus 2 (SARS-CoV-2) by reverse transcription-loop-mediated isothermal amplification. PLoS One 2020; 15: e0234682.

27. Kivelä JM, Jarva H, Lappalainen M, Kurkela S. Saliva-based testing for diagnosis of SARS-CoV-2 infection: a meta-analysis. J Med Virol 2021; 93: 1256-8.

28. Czumbel LM, Kiss S, Farkas N, et al. Saliva as a candidate for COVID-19 diagnostic testing: a meta-analysis. Front Med (Lausanne) 2020; 7: 465 .

29. Riccò M, Ranzieri S, Peruzzi S, et al. RT-qPCR assays based on saliva rather than on nasopharyngeal swabs are possible but should be interpreted with caution: results from a systematic review and metaanalysis. Acta Biomed 2020; 91: e2020025.

30. Butler-Laporte G, Lawandi A, Schiller I, et al. Comparison of saliva and nasopharyngeal swab nucleic acid amplification testing for detection of SARS-CoV-2: a systematic review and meta-analysis. JAMA Intern Med 2021; 181: 353-60.

31. Bastos ML, Perlman-Arrow S, Menzies D, et al. The sensitivity and costs of testing for SARS-CoV-2 infection with saliva versus nasopharyngeal swabs: a systematic review and meta-analysis. Ann Intern Med 2021 2021; 174: 501-10.

32. Bulilete $\mathrm{O}$, Lorente $\mathrm{P}$, Leiva $\mathrm{A}$, et al. Panbio ${ }^{\mathrm{TM}}$ rapid antigen test for SARS-CoV-2 has acceptable accuracy in symptomatic patients in primary health care. J Infect 2021; 82: 391-8.

33. Vogels CBF, Watkins AE, Harden CA, et al. SalivaDirect: a simplified and flexible platform to enhance SARS-CoV-2 testing capacity. medRxiv 2020; Sep 28: https://doi.org/10.1016/j.medj.2020.12.010.
34. Azzi L, Carcano G, Gianfagna F, et al. Saliva is a reliable tool to detect SARS-CoV-2. J Infect 2020; 81: e45-50.

35. Fang Z, Zhang Y, Hang C, et al. Comparisons of viral shedding time of SARS-CoV-2 of different samples in ICU and non-ICU patients. $J$ Infect 2020; 81: 147-78.

36. To KK, Tsang OT, Leung WS, et al. Temporal profiles of viral load in posterior oropharyngeal saliva samples and serum antibody responses during infection by SARS-CoV-2: an observational cohort study. Lancet Infect Dis 2020; 20: 565-74.

37. Williams E, Bond $\mathrm{K}$, Zhang B, et al. Saliva as a noninvasive specimen for detection of SARS-CoV-2. J Clin Microbiol 2020; 58: e00776-20.

38. Hung KF, Sun YC, Chen BH, et al. New COVID-19 saliva-based test: how good is it compared to the current nasopharyngeal or throat swab test? J Chin Med Assoc 2020; 83: 891-4.

39. Procop GW, Shrestha NK, Vogel S, et al. A direct comparison of enhanced saliva to nasopharyngeal swab for the detection of SARS CoV-2 in symptomatic patients. J Clin Microbiol 2020; 58: e01946-20

40. Wong RC, Wong AH, Ho YL-L, et al. Evaluation on testing of deep throat saliva and lower respiratory tract specimens with Xpert Xpress SARS-CoV-2 assay. J Clin Virol 2020; 131: 104593.

41. McCormick-Baw C, Morgan K, Gaffney D, et al. Saliva as an alternate specimen source for detection of SARS-CoV-2 in symptomatic patients using Cepheid Xpert Xpress SARS-CoV-2. J Clin Microbiol 2020; 58 e01109-20.

42. Australian Government Department of Health. Testing Framework for COVID-19 in Australia. 25 Jun 2021; cited 4 July 2021. https://www. health.gov.au/resources/publications/coronavirus-covid-19-testingframework-for-covid-19-in-australia

43. European Centre for Disease Prevention and Control. Considerations on the use of rapid antigen detection (including self-) tests for SARS CoV-2 in occupational settings. 6 May 2021; cited 4 July 2021. https:// www.ecdc.europa.eu/en/publications-data/considerations-use-rapidantigen-detection-including-self-tests-sars-cov-2

44. Masiá M, Fernández-González M, Sánchez M, et al. Nasopharyngeal Panbio COVID-19 antigen performed at point-of-care has a high sensitivity in symptomatic and asymptomatic patients with higher risk for transmission and older age. Open Forum Infect Dis 2021; 8: ofab059.

45. European Centre for Disease Prevention and Control. Methodology for Estimating Point Prevalence of SARS-CoV-2 Infection by Pooled RT PCR Testing. Stockholm: ECDC, 2020.

46. de Wolff T, Pflüger D, Rehme M, Heuer J, Bittner M-I. Evaluation of pool-based testing approaches to enable population-wide screening for COVID-19. arXiv 2020; Oct 8: arXiv:2004.11851.

47. European Centre for Disease Prevention and Control. Options for the Use of rapid antigen tests for COVID-19 in the EU/EEA and the UK. 19 Nov 2020; cited 12 Apr 2021. https://www.ecdc.europa.eu/en/ publications-data/options-use-rapid-antigen-tests-covid-19-eueeaand-uk

48. Centers for Disease Control and Prevention. Interim guidance for use of pooling procedures in SARS-CoV-2 diagnostic, screening, and surveillance testing. 2020; cited 21 Sep 2020. https://www.cdc.gov/ coronavirus/2019-ncov/lab/pooling-procedures.html

49. Chong B, Tran T, Druce J, et al. Sample pooling is a viable strategy for SARS-CoV-2 detection in low-prevalence settings. Pathology 2020; 52: $796-800$

50. Luibke N, Senff $\mathrm{T}$, Scherger $\mathrm{S}$, et al. Extraction-free SARS-CoV-2 detection by rapid RT-qPCR universal for all primary respiratory materials. J Clin Virol 2020; 130: 104579.

51. Michel D, Danzer KM, Groß R, et al. Rapid, convenient and efficien kit-independent detection of SARS-CoV-2 RNA. J Virol Methods 2020; 286: 113965

52. Wee SK, Sivalingam SP, Yap EPH. Rapid direct nucleic acid amplification test without RNA extraction for SARS-CoV-2 using a portable PCR thermocycler. Genes (Basel) 2020; 11: 2020.

53. Peto L, Rodger G, Carter DP, et al. Diagnosis of SARS-CoV-2 infection with LamPORE, a high-throughput platform combining loop-mediated isothermal amplification and nanopore sequencing. medRxiv 2020; Sep 25: https://doi.org/10.1101/ 2020.10.24.20218685.

54. GOV.UK. Roll-out of 2 new rapid coronavirus tests ahead of winter. 3 Aug 2020; cited 20 Sep 2020. https://www.gov.uk/government/news/ roll-out-of-2-new-rapid-coronavirus-tests-ahead-of-winter

55. Bhoyar R, Jain A, Sehgal P, et al. High throughput detection and genetic epidemiology of SARS-CoV-2 using COVIDSeq next generation sequencing. bioRxiv 2020; Aug 10: https://doi.org/10.1101/ 2020.08.10.242677.

56. US Food and Drug Administration. Emergency use authorization (EUA) summary for Helix COVID-19 NGS test. Cited 12 Apr 2021. https://www.fda.gov/media/140917/download 
57. Fenollar F, Bouam A, Ballouche M, et al. Evaluation of the Panbio COVID-19 rapid antigen detection test device for the screening of patients with COVID-19. J Clin Microbiol 2021; 59: e02589-20.

58. Merino P, Guinea J, Muñoz-Gallego I, et al. Multicenter evaluation of the Panbio ${ }^{\mathrm{TM}}$ COVID-19 rapid antigen-detection test for the diagnosis of SARS-CoV-2 infection. Clin Microbiol Infect 2021; 27: 758-61.

59. Torres I, Poujois S, Albert E, Colomina J, Navarro D. Evaluation of a rapid antigen test (Panbio ${ }^{\mathrm{TM}}$ COVID-19 Ag rapid test device) for SARS-CoV-2 detection in asymptomatic close contacts of COVID-19 patients. Clin Microbiol Infect 2021; 27: 636.e1-4.

60. Albert E, Torres I, Bueno F, et al. Field evaluation of a rapid antigen test (Panbio ${ }^{\mathrm{TM}}$ COVID-19 Ag Rapid Test Device) for COVID-19 diagnosis in primary healthcare centres. Clin Microbiol Infect 2021; 27: $472 . e 7-10$

61. Gremmels H, Winkel B, Shuurman R, et al. Real-life validation of the Panbio ${ }^{\mathrm{TM}}$ COVID-19 antigen rapid test (Abbott) in community dwelling subjects with symptoms of potential SARS-CoV-2 infection. EClinicalMedicine 2021; 31: 100677.

62. Linares M, Pérez-Tanoira R, Carrero A, et al. Panbio antigen rapid test is reliable to diagnose SARS-CoV-2 infection in the first 7 days after the onset of symptoms. J Clin Virol 2020; 133: 104659.

63. González-Donapetry P, García-Clemente P, Bloise I, et al. Think of the children: evaluation of SARS-CoV-2 rapid antigen test in pediatric population. Pediatr Infect Dis J 2021; 40: 385-8.

64. Lee RA, Herigon JC, Benedetti A, et al. Performance of saliva, oropharyngeal swabs, and nasal swabs for SARS-CoV-2 molecular detection: a systematic review and meta-analysis. J Clin Microbiol 2021; 59: e02881-20.

65. Berger A, Nsoga MTN, Perez-Rodriguez FJ, et al. Diagnostic accuracy of two commercial SARS-CoV-2 antigen-detecting rapid tests at the point of care in community-based testing centers. PLoS One 2021; 16 e0248921.

66. Villaverde S, Domínguez-Rodríguez S, Sabrido G, et al. Diagnostic accuracy of the Panbio severe acute respiratory syndrome coronavirus 2 antigen rapid test compared with reverse-transcriptase polymerase chain reaction testing of nasopharyngeal samples in the pediatric population. J Pediatr 2021; 232: 287-9.e4.

67. Cerutti F, Burdino E, Milia MG, et al. Urgent need of rapid tests for SARS CoV-2 antigen detection: evaluation of the SD-Biosensor antigen test for SARS-CoV-2. J Clin Virol 2020; 132: 104654.

68. Barker A. Could a breathalyser help detect COVID-19? Indonesian scientists say they've developed one. 11 Jan 2021; cited 12 Apr 2021. https:/ www.abc.net.au/news/2021-01-02/indonesia-to-use-breathalysers-tohelp-detect-covid-19/13019744

69. Muhi S, Tayler N, Hoang T, et al. Multi-site assessment of rapid, pointof-care antigen testing for the diagnosis of SARS-CoV-2 infection in a low-prevalence setting: a validation and implementation study. Lancet Reg Health West Pac 2021; 9: 100115.

70. Krüttgen A, Cornelissen CG, Dreher M, Hornef MW, Imöhl M, Kleines M. Comparison of the SARS-CoV-2 rapid antigen test to the real star Sars-CoV-2 RT PCR kit. J Virol Methods 2021; 288: 114024.

71. Lindner AK, Nikolai O, Kausch F, et al. Head-to-head comparison of SARS-CoV-2 antigen-detecting rapid test with self-collected nasal swab versus professional-collected nasopharyngeal swab. Eur Respir J 2021: 57: 2003961

72. Salvagno GL, Gianfilippi G, Bragantini D, Henry BM, Lippi G. Clinical assessment of the Roche SARS-CoV-2 rapid antigen test. Diagnosis (Berl) 2021; Jan 18: https://doi.org/10.1515/dx-2020-0154.

73. Chaimayo C, Kaewnaphan B, Tanlieng N, et al. Rapid SARS-CoV-2 antigen detection assay in comparison with real-time RT-PCR assay for laboratory diagnosis of COVID-19 in Thailand. Virol J 2020; 17: 177.

74. Mak GC, Lau SS, Wong KK, et al. Analytical sensitivity and clinical sensitivity of the three rapid antigen detection kits for detection of SARS-CoV-2 virus. J Clin Virol 2020; 133: 104684.

75. Nalumansi A, Lutalo T, Kayiwa J, et al. Field evaluation of the performance of a SARS-CoV-2 antigen rapid diagnostic test in Ugand using nasopharyngeal samples. Int J Infect Dis 2021; 104: 282-6.

76. Jääskeläinen $\mathrm{AE}$, Ahava MJ, Jokela $\mathrm{P}$, et al. Evaluation of three rapid lateral flow antigen detection tests for the diagnosis of SARS-CoV-2 infection. J Clin Virol 2021; 137: 104785.

77. Gootenberg JS, Abudayyeh OO, Lee JW, et al. Nucleic acid detection with CRISPR-Cas13a/C2c2. Science 2017; 356: 438-42.

78. Joung J, Ladha A, Saito M, et al. Detection of SARS-CoV-2 with SHERLOCK one-pot testing. N Engl J Med 2020; 383: 1492-4.

79. Huang Z, Tian D, Liu Y, et al. Ultra-sensitive and high-throughput CRISPR-powered COVID-19 diagnosis. Biosens Bioelectron 2020; 164: 112316

80. Mustafa MI, Makhawi AM. Sherlock and Detectr: CRISPR-Cas systems as potential rapid diagnostic tools for emerging infectious diseases. J Clin Microbiol 2021; 59: e00745-20.
81. KeyOptions. Virolens. Cited 18 Sep 2020. https://virolens.keyoptions.

82. Wikipedia. Coronavirus breathalyzer. Cited 12 Apr 2021. https://en wikipedia.org/wiki/Coronavirus_breathalyzer

83. Globe Jakarta. Indonesia's AI-powered Covid-19 test GeNose cleared for public use. 26 Dec 2020; cited 12 Apr 2021. https://jakartaglobe. $\mathrm{id} /$ tech/indonesias-aipowered-covid19-test-genose-cleared-forpublic-use

84. National Institutes of Health. NIH to support radical approaches to nationwide COVID-19 testing and surveillance. 21 Dec 2020; cited 12 Apr 2021. https://www.nih.gov/news-events/news-releases/nihsupport-radical-approaches-nationwide-covid-19-testing-surveillance

85. Inquirer.net. Dutch to roll out rapid breath tests for Covid-19. $27 \mathrm{Feb}$ 2021; cited 12 Apr 2021. https://newsinfo.inquirer.net/1400721/dutchto-roll-out-rapid-breath-tests-for-covid-19

86. National Institute for Public Health and the Environment. Dutch to roll out rapid breath tests for Covid-19. Cited 12 Apr 2021. https://www. rivm.nl/node/167431

87. de Vries R, Vigeveno RM, Mulder S, et al. Ruling out SARS-CoV-2 infection using exhaled breath analysis by electronic nose in a public health setting. medRxiv 2021; Feb 16: https://doi.org/10.1101/ 2021.02.14.21251712v1.

88. The Conversation. Indonesian-made COVID-19 breathalyser sensitivity comparable to RT-PCR. 2 Mar 2021; cited 12 Apr 2021. https:/ theconversation.com/indonesian-made-covid-19-breathalyser-sensitivity-comparable-to-rt-pcr-155497

89. McArthur A. One-minute 'game changing' COVID-19 breath test currently being trialled in Dubai. 16 Mar 2021; cited 12 Apr 2021. https://www.mobihealthnews.com/news/emea/one-minute-gamechanging-covid-19-breath-test-currently-being-trialled-dubai

90. 360Dx. NIH awards $\$ 249 \mathrm{M}$ to seven firms for rapid acceleration of COVID-19 diagnostics. 2020; cited 20 Sep 2020. https://www.360dx. com/research-funding/nih-awards-249m-seven-firms-rapidacceleration-covid-19-diagnostics\#.X3KBwS8RqqB

91. International Federation of Clinical Chemistry and Laboratory Medicine. Breath analysis for COVID diagnosis - analyzers. 14 Jan 2021 cited 22 Apr 2021. https://www.ifcc.org/media/478809/wg-volanalyzers-2021.pdf

92. Health Sciences Authority. HSA grants provisional authorization for 'Brefence Go COVID-19 Breath Test System' and 'TracieX Breathalyser' for detection of COVID-19 infection. 11 Jun 2021; cited 4 July 2021. https://www.hsa.gov.sg/announcements/regulatory-updates/hsagrants-provisional-authorisation-for-brefence-go-covid-19-breath-testsystem-and-traciex-breathalyser-for-detection-of-covid-19-infection

93. De Vries R, Vigeveno R, Mulder S, et al. Ruling out SARS-CoV-2 infection using exhaled breath analysis by electronic nose in a public health setting. medRxiv 2021; Feb 16: https://doi.org/10.1101/ 2021.02.14.21251712v1.

94. Chromatography Today. Can a breathalyser detect COVID-19? 16 Jun 2021; cited 4 July 2021. https://www.chromatographytoday.com/news/ gc-ms/46/breaking-news/can-a-breathalyser-detect-covid-19/55610

95. World Health Organization. WHO R\&D Blueprint. Consultation on the Use of Trained Dogs for Screening COVID-19 Cases. Geneva: WHO, 2021. https://cdn.who.int/media/docs/default-source/blue-print/ who-consultation-screening-dogs-8th-march-20215d9ed791-3201 $4 \mathrm{e} 3 \mathrm{c}-93 \mathrm{c} 1-\mathrm{a} 846910 \mathrm{~d} 53 \mathrm{cc}$.pdf?sfvrsn=202ce0a_1\&download=true

96. World Health Organization. Status of environmental surveillance for SARS-CoV-2 virus: scientific brief. 7 Aug 2020; cited 4 July 2021. https://www.who.int/news-room/commentaries/detail/status-ofenvironmental-surveillance-for-sars-cov-2-virus

97. O'Reilly KM, Allen DJ, Fine P, et al. The challenges of informative wastewater sampling for SARS-CoV-2 must be met: lessons from polio eradication. Lancet Microbe 2020; 1: e189-90.

98. Ahmed W, Angel N, Edson J, et al. First confirmed detection of SARSCoV-2 in untreated wastewater in Australia: a proof of concept for the wastewater surveillance of COVID-19 in the community. Sci Total Environ 2020; 728: 138764

99. Dietz L, Constant D, Fretz, et al. Exploring integrated environmenta viral surveillance of indoor environments: a comparison of surface and bioaerosol environmental sampling in hospital rooms with COVID-19 patients. medRxiv 2021; Mar 26: https://doi.org/10.1101/ 2021.03.26.21254416.

100. Goods Administration Therapeutics. Post-market evaluation of serologybased point of care tests. Oct 2020; cited 21 Sep 2020. https://www.tga. gov.au/post-market-evaluation-serology-based-point-care-tests

101. Torjesen I. Covid-19: how the UK is using lateral flow tests in the pandemic. BMJ 2021; 372: n287.

102. Wise J. Covid-19: lateral flow tests miss over half of cases, Liverpool pilot data show. BMJ 2020; 371: m4848.

103. Holt E. COVID-19 testing in Slovakia. Lancet Infect Dis 2021; 21: 32. 
104. Pavelka M, Van-Zandvoort K, Abbott S, et al. The impact of population-wide rapid antigen testing on SARS-CoV-2 prevalence in Slovakia. Science 2021; Mar 23: eabf9648.

105. Mays JA, Greninger AL, Jerome KR, et al. Preprocedural surveillance testing for SARS-CoV-2 in an asymptomatic population in the Seattle region shows low rates of positivity. J Clin Microbiol 2020; 58. e01193-20.

106. European Centre for Disease Prevention and Control. COVID-19 testing strategies and objectives. 15 Sep 2020; cited 12 Apr 2021. https://www.ecdc.europa.eu/sites/default/files/documents/ TestingStrategy_Objective-Sept-2020.pdf

107. Ministry of Health, Singapore. Pre-event testing (PET). 24 March 2021; cited 12 Apr 2021. https://www.moh.gov.sg/covid-19/pet

108. Mina MJ, Peto TE, García-Fiñana M, Semple MG, Buchan IE. Clarifying the evidence on SARS-CoV-2 antigen rapid tests in public health responses to COVID-19. Lancet 2021; 397: 1425-7.

109. World Health Organization (WHO). SARS-CoV-2 Antigen-Detecting Rapid Diagnostic Tests: An Implementation Guide. Geneva: WHO, 2020. https://www.who.int/publications/i/item/9789240017740

110. Soleimani R, Deckers C, Huang T, et al. Rapid COVID-19 antigenic tests: usefulness of a modified method for diagnosis. J Med Virol 2021; May 19: https://doi.org/10.1002/jmv.27094.

111. Creager HM, Cabrera B, Schnaubelt A, et al. Clinical evaluation of the BioFire ${ }^{\circledR}$ respiratory panel 2.1 and detection of SARS-CoV-2. J Clin Virol 2020; 129: 104538.

112. Gibani M, Toumazou C, Sohbati M, et al. Assessing a novel, lab-free, point-of-care test for SARS-CoV-2 (CovidNudge): a diagnostic accuracy study. Lancet Microbe 2020; 1: e300-7.

113. Pekosz A, Parvu V, Li M, et al. Antigen-based testing but not real-time polymerase chain reaction correlates with severe acute respiratory syndrome coronavirus 2 viral culture. Clin Infect Dis 2021; Jan 20: ciaa1706.
114. Kilic A, Hiestand B, Palavecino E. Evaluation of performance of the BD Veritor SARS-CoV-2 chromatographic immunoassay test in patients with symptoms of COVID-19. J Clin Microbiol 2021; 59 e00260-21.

115. Pollock NR, Tran K, Jacobs JR, et al. Performance and operational evaluation of the Access Bio CareStart rapid antigen test in a highthroughput drive-through community testing site in Massachusetts. medRxiv 2021; Mar 9: https://doi.org/10.1101/2021.03.07.21253101.

116. Rottenstreich A, Zarbiv G, Kabiri D, et al. Rapid antigen detection testing for universal screening for severe acute respiratory syndrome coronavirus 2 in women admitted for delivery. Am J Obstet Gyneco 2021; 224: 539-40.

117. González-Donapetry P, García-Clemente P, Bloise I, et al. Think of the children. Evaluation of SARS-CoV-2 rapid antigen test in pediatric population. Pediatr Infect Dis J 2021; 40: 385-8.

118. Jääskeläinen $\mathrm{AE}$, Ahava $\mathrm{MJ}$, Jokela $\mathrm{P}$, et al. Evaluation of three rapic lateral flow antigen detection tests for the diagnosis of SARS-CoV-2 infection. J Clin Virol 2021; 137: 104785.

119. Kruttgen A, Cornelissen C, Dreher M, et al. Comparison of the SARS CoV-2 rapid antigen test to the real star Sars-CoV-2 RT PCR kit. I Virol Methods 2021; 288: 114024

120. Beck ET, Paar W, Fojut L, Serwe J, Jahnke RR. Comparison of the Quidel Sofia SARS FIA test to the Hologic Aptima SARS-CoV-2 TMA test for diagnosis of COVID-19 in symptomatic outpatients. J Clin Microbiol 2021; 59: e02727-20.

121. Pray IW, Ford L, Cole D, et al. CDC COVID-19 Surge Laboratory Group. Performance of an antigen-based test for asymptomatic and symptomatic SARS-CoV-2 testing at two university campuses Wisconsin, September-October 2020. MMWR Morb Mortal Wkly Rep 2021; 69: 1642-7. 